\title{
Editorial
}

\section{SCOPE OF BARIATRIC/METABOLIC SURGERY IN BANGLADESH}

Bariatric surgery started its journey in the western world towards the last quarter of the last century because obesity as a public health problem was almost an exclusive preserve of the affluent western world. It had mortality and significant morbidity in the early years of bariatric surgery as a result of it's inadequately understood and poorly managed metabolic consequences. Inappropriate selection of patients also contributed to this morbidity. Bariatric surgery was initially done as open procedures. Subsequently it was performed laparoscopically. Fortunately for the surgeons and the patients it has evolved into its modern safe stage.

Bariatric procedures can be restrictive where the amount of food intake can be reduced. Alternatively, it can be malabsorptive where absorption of food will be reduced. Both the procedures may be combined with double effects of both restriction and malabsorption. Permanent restrictive procedure like gastric sleeve resection additionally does produce its effect of weight reduction via hormonal mechanism, gastric hormone ghrelin playing an important role. Temporary restrictive procedures include endoscopic insertion of intragastric balloon or laparoscopic placement of adjustable gastric bands. Malabsorptive procedures include Rou- en- $Y$ gastric bypass or Duodenal switch operations.

Bariatric surgery is also called Metabolic surgery as it deals with metabolic syndrome which comprises diabetes, hypertension, dyslipidaemia, and sleep apnea in addition to induction of excessive body weight loss.

Before deciding on Bariatric surgery one has to know Body Mass Index (BMI). BMI is worked out as body weight in $\mathrm{Kg}$. divided by height in Metres squared (Wt.in Kg/Ht.in Metres ${ }^{2}$ ) A normal person should have a BMI between 20 and 25. A person with a BMI of 2530 is overweight. Body Mass Index between 30-40 denotes obesity. BMI greater than 40 implies morbid obesity and that greater than 50 means super obesity.

Metabolic surgery is indicated in morbidly obese and super obese patients. Metabolic surgery positively impacts coronary heart disease ${ }^{1}$. It is also indicated in obese patients with various elements of metabolic syndrome. $80 \%$ of patients are cured of their associated diabetes and hypertension after surgery without needing any medications while others need significantly reduced doses of medication. ${ }^{2}$

There is no valid data about the obesity amongst normal population in our country. Recent survey carried out by The Daily Star amongst school children showed one in ten children is obese probably due to unhealthy food habits of the affluent section of children. Extrapolating from this data one can indulge in an educated guess that significant \% of population in Bangladesh suffer from obesity of different types. These obese peoples are likely to have associated co-morbid conditions like hypertension and diabetes. There should be public awareness campaign against obesity and its impact on health. Educated, informed and well to do section of the people have already started moving out of the country to have anti -obesity surgery done.

There should be an effort on the part of public health department to carry out a survey for creation of a data base on obesity in our country. Government should open Metabolic/Bariatric Surgery Unit in tertiary care hospital in Dhaka with well trained surgeons, surgical team and nutritionist who can cater for the need of our people. This will prevent exodus of patients with obesity into the neighbouring country putting a big drain on our hard earned foreign exchange.

M.A. Majid, FRCS, FCPS.

Professor of Surgery (Retired), House 14, Road 68, Gulshan 2, Dhaka, E-mail>profmamajid@gmail.com,

\section{References:}

1. Nguyen NT, Goldman C, Rosenquist C et al, Laparoscopic Versus Open Gastric Bypass: A Randomized Study of Outcomes, Quality of Life and Costs. Annals of Surgery, 2001;.234(3): 279-291.

2. Harvey J, Sugerman LG. Wolfe, Domenic A. Sica et al. Diabetes and Hypertension in Severe Obesity and Effects of Gastric Bypassed-Induced Weight Loss. Annals of Surgery, 2003; 237(6): 751-758. 